\title{
On diffusion, drift and the Einstein relation
}

\author{
S E Guidoni and C M Aldao
}

Institute of Materials Science and Technology (INTEMA), Universidad Nacional de Mar del Plata-CONICET, Juan B. Justo 4302, 7600 Mar del Plata, Argentina

Received 7 November 2001, in final form 8 April 2002

Published 17 June 2002

Online at stacks.iop.org/EJP/23/395

\begin{abstract}
The diffusivity, mobility and limitations of the Einstein relation are discussed by means of two pedagogical examples. In a simple one-dimensional analysis, we compute the consequences of the single-particle occupancy restriction and correlations over two successive steps on the diffusion and drift fluxes and on the particle distribution functions.
\end{abstract}

\section{Introduction}

Diffusion and drift are topics included in many courses not only for physicists but also for engineers and material scientists [1-4]. In this work we focus on two pedagogical examples that show how to solve apparently difficult problems. Also, with these models, we emphasize that the Einstein relation must not be assumed to hold outside its domain of validity.

Diffusion in diverse media has been widely studied since the pioneering work of Fick [5]. He proposed that diffusion of matter obeys a phenomenological law of the same general form as Fourier's law of heat conduction. What is now generally known as Ficks's first law of diffusion defines the diffusivity or diffusion constant $D$ (we consider here only the one-dimensional case),

$$
J_{D}=-D \frac{\partial c}{\partial x}
$$

where $J_{D}$ is the flux of diffusing particles and $c$ its concentration.

An atomistic approach to diffusion is usual in textbooks where the equivalence between a random walk and diffusion is exploited. Random walkers that do not interfere with each other and step with equal probability in any direction are regular assumptions. At some point, the physics teacher should not avoid addressing the distinction between the two approaches to diffusion: mass transport resulting from a concentration gradient and Brownian motion of individual diffusants (see [6] for a detailed and comprehensive treatment). Diffusion coefficients derived in both ways can contain possible effects of interactions between particles and can depend on particle concentration. The two approaches need only give the same result in the zero-concentration limit. 
If, as a consequence of an external force $F$ particles with a concentration $c$ move with an average velocity $v$, then the resulting flux is $J_{F}=c v$. Defining the mobility $\mu$ as the ratio between average velocity and force, the drift flux can be expressed as

$$
J_{F}=\mu c F \text {. }
$$

The so-called Einstein relation links the mobility to the diffusion constant. This relation is usually derived considering that the total particle flux or current must vanish in thermal equilibrium [7-11]. Indeed, if the total current is null, then

$$
\mu c F-D \frac{\partial c}{\partial x}=0
$$

This equation can be expressed as [12]

$$
\mu=-\frac{D}{c} \frac{\partial c}{\partial V}
$$

where it was considered that $F=-\partial V / \partial x$. If the particle density is given by the Boltzmann distribution, i.e. $c \sim \exp \left[-V(x) / k_{B} T\right]$, it is easy to show from equation (3) that the mobility and diffusion constant are directly related as

$$
D=k_{B} T \mu \text {. }
$$

Equation (5) is known as the Einstein relation.

In most systems, particles interact and then the analysis of diffusion and drift currents becomes complicated because jumping probabilities depend on the local environment of the particle. There are also many situations in which the microscopic movement of particles present correlations implying a 'memory' of previous events. Thus, although following a random walk movement, particles may tend to keep moving in the same direction as the previous jump (persistence) or in the opposite direction as the previous jump (antipersistence). Then, we will examine two cases, one presenting particle interaction (lattice gas model) and the other with particle correlation. Consequences for diffusion and drift will be determined.

\section{Multiple- and single-particle occupancy}

Equation (1) can be easily derived with an atomistic approach for multiple occupancy in a one-dimensional lattice. Consider two adjacent sites separated by a distance $a$ as shown in figure 1. Let us suppose that $n_{1}$ is the number of particles at site 1 and $n_{2}$ at site 2 . Particles are restricted to jumps between nearest-neighbour positions and do not have any kind of interaction between themselves. Let us now define a quantity $\gamma$, the mean jump frequency, that is, the average number of times that a particle jumps in any one direction, that is to the right and to the left. Then, the flux of particles moving from site 1 to site 2 is $\gamma n_{1}$ and from site 2 back to site 1 is $\gamma n_{2}$. Therefore, the net flux from site 1 to site 2 is given by

$$
J_{D}=J_{1}-J_{2}=-\gamma\left(n_{2}-n_{1}\right),
$$

and in terms of concentration $c$

$$
J_{D}=-\gamma a^{2} \frac{\left(c_{2}-c_{1}\right)}{a} .
$$

Finally, passing to the limit, equation (7) gives us

$$
J_{D}=-\gamma a^{2} \frac{\partial c}{\partial x}=-D \frac{\partial c}{\partial x} .
$$

From equation (8) the diffusion coefficient can be expressed in terms of the mean jump frequency

$$
D=\gamma a^{2}
$$




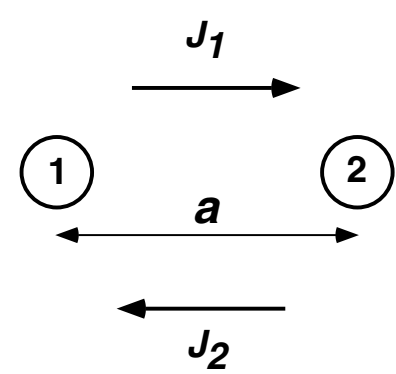

Figure 1. Representation of two sites and associated fluxes.

Next, we will analyse a similar problem in which the jump will succeed only if it is directed towards a vacant site, that is, we only allow single occupancy. This occurs when there is a short-range repulsive interaction between adjacent particles. As expected, fluxes are directly affected by this restriction; now

$$
J_{1}=\gamma f_{1}\left(1-f_{2}\right), \quad J_{2}=\gamma f_{2}\left(1-f_{1}\right),
$$

where $f$ is the occupancy probability, with values of either 0 or 1 . Note that in this case $\gamma$ represents the attempt-to-jump frequency. The net flow can be written as

$$
J_{D}=J_{1}-J_{2}=-\gamma\left(f_{2}-f_{1}\right),
$$

which is equivalent to equation (6) and then the final result given in equation (9) can be derived. Therefore, the resulting diffusivity is the same, that is $\gamma a^{2}$, as when multiple occupancy is allowed. It is clear that the probability of a successful jump decreases with occupancy probability, as seen in equations (10), but it is remarkable that the diffusivity remains constant. This can be understood since particle movement probabilities are equally reduced in both directions.

The next step is to determine the mobility in both cases, when any number of particles per site is allowed and when only single occupancy is allowed. We will consider a small potential gradient and then a small force acting on the particles. The situation is depicted in figure 2 where we have considered that the applied potential gradient acts to increase the jumping frequency towards the right by a factor $p$. (The same final result is obtained if the applied potential gradient is considered to reduce the jumping towards the left or a combination of both effects.) $p$ can be expressed as

$$
p=\exp \left(\Delta V / k_{B} T\right) \approx 1+\Delta V / k_{B} T .
$$

For multiple occupancy we can write

$$
J_{F}=n_{1} \gamma p-n_{2} \gamma .
$$

We are now interested in the drift current with no diffusion present. Then, we assume $n_{1}=n_{2}=n$ and using equation (12) the drift current takes the form

$$
J_{F}=n \gamma \frac{\Delta V}{k_{B} T}
$$

In terms of concentration $c$, equation (14) becomes

$$
J_{F}=\frac{c a^{2} \gamma}{k_{B} T} \frac{\Delta V}{a} .
$$

Finally, expressing $\Delta V / a$ as the force over the particle, equation (15) can be written as

$$
J_{F}=\frac{\gamma a^{2} c F}{k_{B} T} .
$$




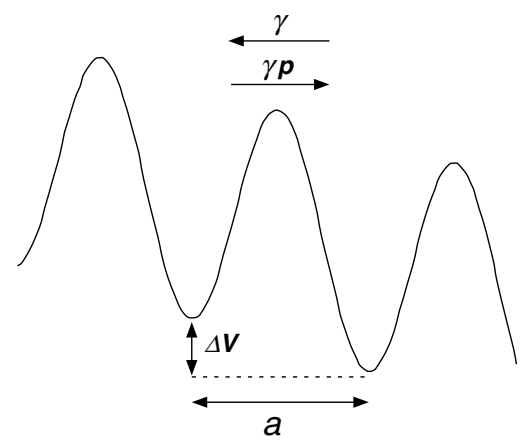

Figure 2. Schematic energy barrier diagram in the presence of a potential gradient.

By comparison with equation (2), the mobility is given by

$$
\mu=\frac{\gamma a^{2}}{k_{B} T} .
$$

From equations (9) and (17), it is apparent that the Einstein relation is valid if multiple occupancy is allowed.

Finally, let us determine the mobility when only single occupancy is allowed. As in determining the diffusivity, we will consider $f$ to be the occupancy. The drift current can be expressed as

$$
J_{F}=f_{1}\left(1-f_{2}\right) \gamma p-f_{2}\left(1-f_{1}\right) \gamma
$$

Following the same steps used in deriving equation (16), equation (18) can be easily expressed as

$$
J_{F}=\frac{c(1-f) \gamma a^{2} F}{k_{B} T} .
$$

By comparing with equation (2), the mobility is

$$
\mu=\frac{\gamma a^{2}(1-f)}{k_{B} T} \text {. }
$$

Clearly, the effect of the excluded volume consists of directly reducing the mobility. This simple analysis shows that the Einstein relation is not valid since particles limited to single occupation are not expected to obey the Boltzmann distribution.

With the diffusivity and mobility found for the single-occupancy case, equation (4) becomes a differential equation for the particle concentration. Then, after integration $f$ can be expressed as a function of the potential

$$
f=\frac{1}{1+\exp \left[\left(V-V_{0}\right) / k_{B} T\right]}
$$

where $V_{0}$ is a constant that depends on the number of particles. Interestingly, equation (21) resembles the Fermi-Dirac distribution function.

If no field is applied, the evolution of an excess particle concentration pulse is the same for multiple or single occupancy since the diffusivity is the same in both cases. By approximating the initial pulse with an impulse, the shape of the excess carrier pulse can be described analytically as a spreading Gaussian curve. When a constant field is applied and multiple occupancy is allowed, the pulse shape is not affected and the spreading Gaussian curve travels with a speed that depends directly on the field strength and the mobility.

For single occupancy, due to the dependence of the mobility on the concentration, see equation (19), an external force can affect the pulse evolution in a more complicated manner. 
Indeed, the mobility depends on the concentration and then the pulse deforms as it travels. Since the mobility decreases with occupancy the ends of the pulse travel faster than the centre and consequently the pulse becomes asymmetric. The resulting differential equation for single occupancy is similar to the Burgers equation. The time development of the solution of this equation can be found in [13] where the asymmetric form of the resulting profile is shown.

\section{Random walk with correlated jumps}

In correlated random walks particles have 'memory' in the sense that a jump depends on previous ones. As a particularly simple example, we will consider correlations over two successive jumps. In the standard procedure, to determine diffusion coefficients in periodic lattices, appropriate master equations are established and through a Fourier expansion an eigenvalue problem is solved [14]. Eigenvalues are then calculated and the diffusion coefficient determined. Here we will use an alternative approach to determine the diffusion coefficients, not regularly applied in the literature, that simplifies the calculations [15]. With this method this problem can be solved using algebra only.

Particles are characterized by two properties: the position given by an integer number that labels the site where particles sit, and a plus or minus sign indicating where particles arrived from. Particles cannot jump over more than one site, i.e. they can only jump to nearestneighbour sites. Accordingly, the number of particles at site $i$ is split into two contributions, $n_{i}^{+}$ and $n_{i}^{-}$, where + and - indicate that the particle came from the sites $i+1$ or $i-1$, respectively. The rate for a jump in the same direction as the previous jump is denoted by $\gamma_{f}$ and the rate for a jump in the opposite direction is denoted by $\gamma_{b}$.

To compute the evolution of the number of particles at a given site $i$, the rates at which particles leave the site and arrive at it must be considered. Hence,

$$
\begin{aligned}
\frac{\mathrm{d} n_{i}^{+}}{\mathrm{d} t} & =n_{i+1}^{+} \gamma_{f}+n_{i+1}^{-} \gamma_{b}-n_{i}^{+}\left(\gamma_{f}+\gamma_{b}\right), \\
\frac{\mathrm{d} n_{i}^{-}}{\mathrm{d} t} & =n_{i-1}^{+} \gamma_{f}+n_{i-1}^{-} \gamma_{b}-n_{i}^{-}\left(\gamma_{f}+\gamma_{b}\right) .
\end{aligned}
$$

Note that for $n_{i}^{+}$there is no contribution from site $i-1$ and for $n_{i}^{-}$there is no contribution from site $i+1$. After some time the system reaches a steady state at which the number of particles in each site remains constant and also particle fluxes (a net flux from right to left will be assumed). In what follows we will show an easy way to determine the diffusivity using Fick's law and relations derived from equations (22) considering the system is under steady state.

A diffusion flux does not directly depend on the number of particles but on how the number of particles changes from site to site, i.e. $\mathrm{d} c / \mathrm{d} x$ (see equation (1)). Therefore, to have a diffusion flux, a gradient in the particle concentration must be present and, since only this gradient is relevant, any arbitrary concentration offset can be chosen. Taking an appropriate concentration offset, we can impose that the flux from a given site to the right be null; this site will be $i=0$ in what follows. This choice implies $n_{0}^{+} \gamma_{b}+n_{0}^{-} \gamma_{f}=0$, a condition that we can satisfy in two ways. First, we can consider that the chain of sites continues to the left. This leads to a negative value for $n_{0}^{-}$, a counter-intuitive result (anyway, if we deal with an infinite chain, at some point the number of particles will become negative). We prefer to adopt a different point of view, namely to satisfy the condition $J_{0}^{+}=0$ as a consequence of having a finite chain with an end at site 0 . This site is considered to be an ideal sink for particles, i.e. $n_{0}^{+}=n_{0}^{-}=0$, which avoids undesirable negative concentrations. On the other end of the chain a reservoir or source of particles is necessary to maintain the flux.

Then, regardless of the interpretation adopted, for the three neighbouring sites depicted in figure 3 we can write 


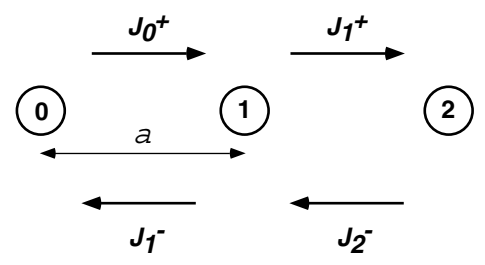

Figure 3. Representation of three sites and associated fluxes.

$$
\begin{array}{ll}
J_{0}^{+}=0, & J_{1}^{-}=n_{1}^{+} \gamma_{f}=n_{1} \gamma_{f}, \\
J_{1}^{+}=n_{1}^{+} \gamma_{b}=n_{1} \gamma_{b}, & J_{2}^{-}=n_{2}^{+} \gamma_{f}+n_{2}^{-} \gamma_{b},
\end{array}
$$

where we have considered that $n_{1}^{+}+n_{1}^{-}=n_{1}$ since $n_{1}^{-}=0$.

As discussed above, under steady-state conditions the number of particles in a site must be constant, then, bearing in mind that site 0 is an ideal sink,

$$
\begin{aligned}
& \frac{\mathrm{d} n_{1}^{+}}{\mathrm{d} t}=n_{2}^{+} \gamma_{f}+n_{2}^{-} \gamma_{b}-n_{1}^{+}\left(\gamma_{f}+\gamma_{b}\right)=0, \\
& \frac{\mathrm{d} n_{2}^{-}}{\mathrm{d} t}=n_{1}^{+} \gamma_{b}-n_{2}^{-}\left(\gamma_{f}+\gamma_{b}\right)=0 .
\end{aligned}
$$

With these equations $n_{2}^{+}$and $n_{2}^{-}$can be expressed as a function of $n_{1}$ as

$$
n_{2}^{-}=\frac{n_{1} \gamma_{b}}{\gamma_{f}+\gamma_{b}}, \quad n_{2}^{+}=\frac{n_{1}\left(\gamma_{f}+2 \gamma_{b}\right)}{\gamma_{f}+\gamma_{b}} \text {. }
$$

The total number of particles at site 2 is calculated as $n_{2}^{+}+n_{2}^{-}$and then the difference between the number of particles at sites 2 and 1 is

$$
n_{2}-n_{1}=n_{1} \frac{2 \gamma_{b}}{\gamma_{f}+\gamma_{b}}
$$

The net flux between sites 1 and 2 can be calculated to be $-\gamma_{f} n_{1}$. (Note that, being in steady state, the net flux is equal to $J_{0}^{+}-J_{1}^{-}$and also equal to $J_{1}^{+}-J_{2}^{-}$.) Finally, using Ficks's first law, i.e. $J=-D\left(n_{2}-n_{1}\right) / a^{2}$, the diffusivity can be determined

$$
D=\frac{a^{2} \gamma_{f}\left(\gamma_{f}+\gamma_{b}\right)}{2 \gamma_{b}}
$$

This is the result obtained using the standard procedure in [14], in which a set of algebraic equations is established by resorting to Fourier and Laplace transformations.

We will now calculate the drift flux for this correlated random walk. With the aid of figure 1 we can visualize the following analysis. We only need to have in mind that $n_{i}^{+}$and $n_{i}^{-}$ refer to particles that came from sites $i+1$ or $i-1$, respectively. As in the previous section we consider that the applied potential gradient acts to facilitate the jumping towards the right by a factor $p$ (see equation (13)). Then, fluxes can be written as

$$
J_{1}=\left(n_{1}^{-} \gamma_{f}+n_{1}^{+} \gamma_{b}\right) p, \quad J_{2}=n_{2}^{+} \gamma_{f}+n_{2}^{-} \gamma_{b} .
$$

Under steady-state conditions, the number of particles at any site does not change, then

$$
\frac{\mathrm{d} n_{1}^{+}}{\mathrm{d} t}=n_{2}^{+} \gamma_{f}+n_{2}^{-} \gamma_{b}-n_{1}^{+}\left(\gamma_{f}+p \gamma_{b}\right)=0
$$

In calculating the drift current we take $n_{1}^{+}=n_{2}^{+}=n^{+}$and $n_{1}^{-}=n_{2}^{-}=n^{-}$because of translational symmetry, then from equation (29) a simple relation connecting $n^{-}$and $n^{+}$is deduced:

$$
n^{-}=p n^{+}
$$


With equations (28) and (30) the net flux can be expressed as

$$
J_{F}=J_{1}-J_{2}=n^{+} \gamma_{f}\left(p^{2}-1\right) .
$$

Finally, using equation (13), $J_{F}$ can be written as

$$
J_{F}=\frac{2 n^{+} \gamma_{f} \Delta V}{k_{B} T}
$$

and, considering that $2 n^{+}$is approximately equal to $n$ and $J_{F}=\mu c F$, the mobility is given by

$$
\mu=\frac{a^{2} \gamma_{f}}{k_{B} T} .
$$

Comparing equations (27) and (33) it is apparent that, except for the limiting case when $\gamma_{f}=\gamma_{b}$ that corresponds to an uncorrelated diffusion, the Einstein relation does not apply. In this example particles are allowed for multiple occupancy, then the self-diffusion and the collective diffusion are identical. All the results described can be easily checked by means of computer simulations using the Monte Carlo method. This is a nice didactic exercise since the analytical solutions are available.

Having the diffusivity and mobility, by means of equation (5) the particle concentration as a function of the potential can be determined. After integration, the concentration $c$ can be expressed as a function of the potential

$$
c=c_{0} \exp \left\lfloor-\frac{2 \gamma_{b}}{\gamma_{f}+\gamma_{b}} V / k_{B} T\right\rfloor,
$$

where $c_{0}$ is a constant that depends on the number of particles. Equation (34) is similar to the Boltzmann distribution with an effective potential consequence of the correlation through the parameters $\gamma_{f}$ and $\gamma_{b}$.

\section{Conclusions}

Diffusion is a very important topic in solid state physics and of extensive application in many fields. The general treatments of the theory of random walks tend to be of a mathematical sophistication demanding advanced knowledge. Consequently, the physics teacher is limited to very simple problems. In this paper, we show how to deal with non-trivial situations in a manner that can be presented at a college level using only algebra and basic calculus. In particular, we determine diffusivity and mobility in two pedagogical examples that also allow us to show that before assuming that the Einstein relation is valid we must check that the system under study is not outside its domain of validity. Furthermore, we determine the right concentration distributions for the examples presented.

\section{Acknowledgments}

This work was partially supported by the National Council for Scientific and Technical Research (CONICET) of Argentina. We gratefully acknowledge stimulating discussions with Daniel R Avalos, Héctor O Mártin and Alberto de la Torre. We also thank one of the reviewers for his/her critical reading of the manuscript and enlightening comments.

\section{References}

[1] Gardiner C W 1997 Handbook of Stochastic Methods (Heidelberg: Springer) ch 8

[2] Van Kampen N G 1981 Stochastic Processes in Physics and Chemistry (Amsterdam: North-Holland) ch 10

[3] Barrett C R, Nix W D and Tetelman A S 1973 The Principles of Engineering Materials (Englewood Cliffs, NJ: Prentice-Hall) ch 5

[4] Borg R J and Dienes G J 1988 An Introduction to Solid State Diffusion (Boston, MA: Academic) ch 1 
[5] See, for example,

Crank J 1975 The Mathematics of Diffusion (Oxford: Oxford University Press) ch 1 and references therein

[6] Gomer R 1990 Diffusion of adsorbates on metal surfaces Rep. Prog. Phys. 53 917-1002

[7] Ashcroft N W and Mermin N D 1976 Solid State Physics (Philadelphia, PA: Saunders) ch 29

[8] Holmes R A 1970 Physical Principles of Solid State Devices (New York: Holt, Rinehart and Winston) ch 5

[9] Hook J R and Hall H E 1991 Solid State Physics (New York: Wiley) section 6.2

[10] Smith R A 1978 Semiconductors (Cambridge: Cambridge University Press) ch 7

[11] Peters J M 1982 Einstein's relation Eur. J. Phys. 3 19-21

[12] Landsberg P T 1981 Einstein and statistical thermodynamics: III. The diffusion-mobility relation in semiconductors Eur. J. Phys. 2 213-19

[13] Vvedensky D D 1993 Partial Differential Equations with Mathematica (Reading, MA: Addison-Wesley)

[14] Haus J W and Kehr K W 1987 Diffusion in regular and disordered lattices Phys. Rep. 150 263-406 ch 4

[15] Aldao C M, Iguain J L and Mártin H O 1996 A steady-state approach to determine diffusion coefficients: the migration of silicon on the $\mathrm{Si}(100)$ surface Surf. Sci. $366483-90$ 